\title{
Identifying the predictors of needling after XEN gel implant
}

\author{
Neha Midha ${ }^{1} \cdot$ Harsha L. Rao $\mathbb{D}^{2} \cdot$ André Mermoud $^{1} \cdot$ Kaweh Mansouri ${ }^{1,3}$
}

Received: 27 February 2018 / Revised: 14 July 2018 / Accepted: 18 July 2018 / Published online: 11 September 2018

(c) The Royal College of Ophthalmologists 2018

\begin{abstract}
Purpose To identify factors associated with probability of needling after XEN implant.

Methods Analysis of data from prospective case series of patients with standalone XEN implant or combined XEN + cataract. Primary outcome was to determine whether postoperative day 1 and week 1 intraocular pressure (IOP), type of glaucoma, and glaucoma severity affected probability and number of needling interventions required.

Results A total of 149 eyes of 113 patients were included in the analysis. In the XEN alone group, mean IOP at day 1 and week 1 was $9.70 \pm 5.43$ and $10.33 \pm 4.41 \mathrm{mmHg}$, respectively. Eyes with lower IOP on day 1 were less likely to require needling (Odds ratio; OR, 1.14; 95\% CI, 1.02-1.28; $p=0.02$ ). A similar association (OR, 1.15; 95\% CI, 1.06-1.26; $p=$ 0.001) was observed between day 1 IOP in and number of needling interventions. In the XEN + cataract group, mean IOPs at day 1 and week 1 were $13.75 \pm 7.52$ and $11.81 \pm 5.36 \mathrm{mmHg}$, respectively. No significant association was noted between early postoperative IOP and probability or number of needling in this group. With IOP $>20 \mathrm{mmHg}$ the probability of needling was $\sim 80 \%$. This number decreased to $35 \%$ if day 1 IOP was $<10 \mathrm{mmHg}$.

Conclusion Postoperative day 1 IOP is a predictor for needling in patients undergoing standalone XEN implantation. These results suggest that surgeons should not wait too long to intervene via needling if IOP in the early postoperative period is in the high teens or above. Type or severity of glaucoma did not influence the probability of needling or number of needling interventions required.
\end{abstract}

\section{Introduction}

Minimally invasive glaucoma surgeries (MIGS) aim to provide a safer and less-invasive alternative to incisional glaucoma surgeries for the control of intraocular pressure (IOP) $[1,2]$. At present, the XEN gel implant (Allergan, Dublin, CA) is the only Food and Drug Administration (FDA)-approved MIGS device that drains into the subconjunctival space and results in a bleb formation. In blebdependent glaucoma surgeries, the long-term control of IOP is limited by scarring at the subconjunctival and episcleral interface [3]. Role of bleb needling with anti-fibrotic agents

Kaweh Mansouri

kwmansouri@gmail.com

1 Glaucoma Research Center, Montchoisi Clinic, Swiss Vision Network, Lausanne, Switzerland

2 Narayana Nethralaya, 63, Bannerghatta Road, Hulimavu, Bangalore, India

3 Department of Ophthalmology, University of Colorado School of Medicine, Denver, CO, USA like mitomycin C (MMC) and 5-fluorouracil has been well established in post trabeculectomy eyes. Needling with antimetabolites is also being used to restore function in failed or failing blebs post XEN implant. Yook et al. [4] reviewed complications associated with MIGS and revealed that needling was the most frequently reported adverse event after XEN implant, with rates varying from 32 to 47\%. Despite the importance of this postoperative intervention, there is a paucity of data on needling after XEN implantation.

The purpose of this report was to identify the factors that are associated with higher probability of needling after XEN implant.

\section{Materials and methods}

A post hoc analysis was performed on data from a previously published prospective interventional study to evaluate the efficacy of XEN gel implant [5]. This study was conducted at the Glaucoma Research Center, Montchoisi Clinic, Swiss Vision Network, Lausanne. The study was approved by the institutional review board and 
Table 1 Baseline characteristics

$\begin{array}{lll}\begin{array}{l}\text { XEN group } \\ \text { (40 eyes of 29 }\end{array} & \begin{array}{l}\text { XEN }+ \\ \text { cataract group } \\ \text { patients) }\end{array} & \begin{array}{l}\text { (109 eyes of } 84 \\ \text { patients })\end{array} \\ & \end{array}$

\begin{tabular}{llll}
\hline Age (in years) & & & \\
Mean \pm SD & $74.7 \pm 11.4$ & $74.3 \pm 8.6$ & 0.311 \\
Range & $52-95$ & $50-93$ & \\
Male:female & $01: 02.6$ & $01: 02.5$ & 0.913 \\
$\begin{array}{l}\text { Glaucoma diagnosis, n (\%) } \\
\text { POAG }\end{array}$ & $15(51.7 \%)$ & $46(54.8 \%)$ & 0.927 \\
PEXG & $10(34.5 \%)$ & $32(38.1 \%)$ & \\
$\begin{array}{l}\text { Median (IQR) mean } \\
\text { deviation, in decibels }\end{array}$ & $7.1(3.0,12.7)$ & $6.0(3.4,11.1)$ & 0.81 \\
$\begin{array}{l}\text { Median (IQR) average } \\
\text { RNFL thickness, in }\end{array}$ & $69(61,80)$ & $81(70,89)$ & 0.007 \\
microns & & & \\
$\begin{array}{l}\text { Day 1 IOP (in mmHg) } \\
\text { Week 1 IOP (in mmHg) }\end{array}$ & $9.70 \pm 5.43$ & $13.75 \pm 7.52$ & 0.001 \\
\hline
\end{tabular}

$S D$ standard deviation, $P O A G$ primary open-angle glaucoma, $P E X G$ pseudoexfoliative glaucoma, IQR interquartile range, $R N F L$ retinal nerve fiber layer, IOP Intraocular pressure

informed consent was obtained from each patient. The study adhered to the tenets of the Declaration of Helsinki. The study population, study device, surgical techniques, and evaluation methods have been described previously [5]. Only the aspects pertinent to the current study will be described here. In brief, patients with open-angle glaucoma (OAG) were prospectively enrolled from January 2015 to June 2016 and underwent XEN alone or XEN + cataract as decided by the treating physician. The results on efficacy, success rate, and adverse events at 1 year have already been published [5].

\section{Study population}

The eligibility criteria for XEN implantation were as follows: age 18 years or above with a diagnosis of primary or secondary OAG, uncontrolled IOP, progressing glaucoma, and/or intolerance to IOP-lowering drops. Eyes were classified as glaucomatous if they had repeatable (two consecutive) abnormal standard automated perimetry test results (Octopus, Haag Streit, Koeniz, Switzerland) in the presence of abnormal appearing optic discs (presence of neuroretinal rim thinning or localized or diffuse retinal nerve fiber layer (RNFL) defects indicative of glaucoma) by slit lamp assessment or spectral domain optical coherence tomography. Visual field exams required reliability indices better than $15 \%$. Patients with the history of any previous glaucoma surgery were excluded from the study.

\section{Surgical technique}

The procedure was done in the operative room and under the surgical microscope to obtain better visualization of the XEN implant and minimize the risk of damaging it. Topical anesthetic drops were instilled in the eye together with two drops of povidone-iodine $5 \%$ and sterile speculum was inserted. A superior corneal bridle suture was used to expose the bleb area with XEN gel stent for easy manipulation. A bent 27-gauge hypodermic needle was inserted temporally into the subconjunctival space and slowly steered toward the XEN implant. Episcleral adhesions below and above the device were released using sweeping movements taking care not to pull on the device or damage it. At the end of needling procedure, MMC $0.01 \%$ was injected superotemporally in the subconjunctival space and milked posterior to the XEN gel stent.

\section{Outcome measures}

To determine whether early postoperative IOP (day 1 and week 1), age, type of glaucoma, glaucoma severity, and number of preoperative anti-glaucoma medications affected the probability of needling number of needling interventions required. Glaucoma severity was determined using visual field mean deviation (MD) (Octopus, Haag Streit, Koeniz, Switzerland) and average RNFL thickness (Spectralis OCT, Heidelberg Engineering AG, Germany).

\section{Statistical analysis}

Descriptive statistics included mean and standard deviation for normally distributed variables and median and interquartile range for non-normally distributed variables. Categorical variables were described using percentages. Logistic regression models were used to evaluate the associations of early postoperative IOP, age, type of glaucoma, glaucoma severity, and number of preoperative antiglaucoma medications with the probability of needling. Ordered logistic regression models were used to evaluate the associations between the above-mentioned factors and the number of needling procedures required. Results are documented as Odds ratio (OR). $p$ value $<0.05$ was considered statistically significant. All statistical analyses were performed with commercially available software (Stata version 13.1; StataCorp, College Station, TX).

\section{Results}

A total of 149 eyes of 113 glaucoma patients were included in the analysis (40 eyes of 29 patients in XEN alone group and 109 eyes of 84 patients in XEN + cataract group). The 
Fig. 1 a Graph showing the association of probability of needling (with 95\% confidence intervals in dashed lines) with postoperative IOP on day 1 in XEN alone group. b Graph showing the association of probability of needling (with 95\% confidence intervals in dashed lines) with postoperative IOP on day 1 in XEN + cataract group a

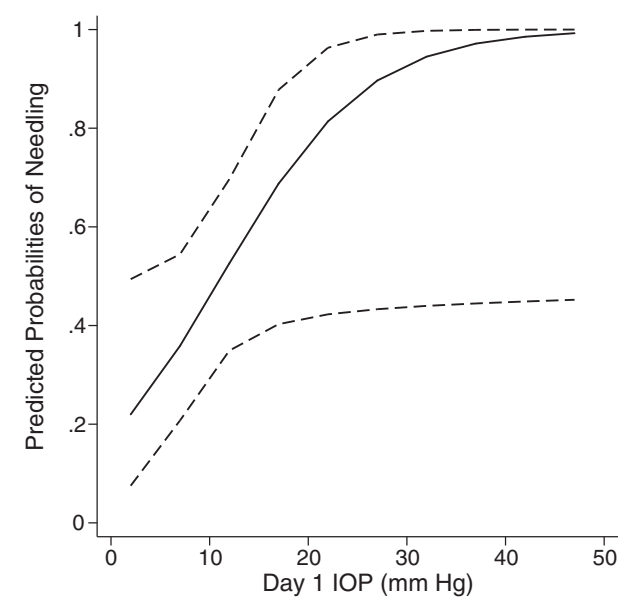

b

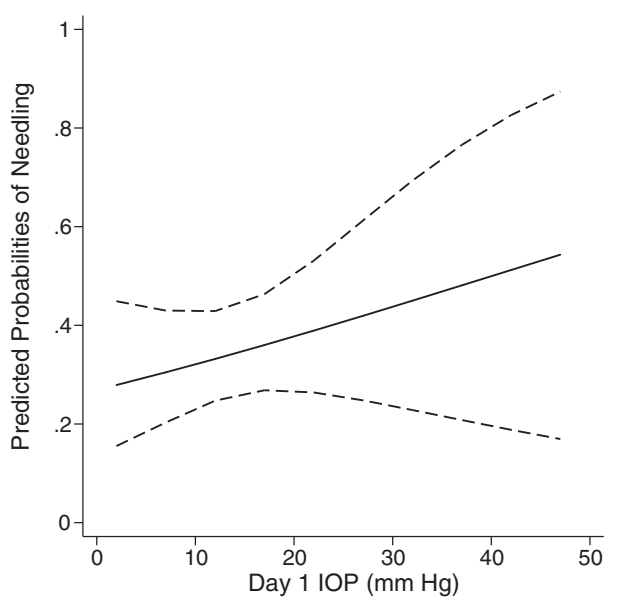

average age was $74.4 \pm 9.4$ years; $71.7 \%$ were female. Most patients were Caucasian $(96.5 \%)$ and had primary openangle glaucoma (POAG; 54.0\%) or pseudoexfoliative glaucoma (PEXG; 37.2\%). The mean pre needling IOP in XEN alone and in XEN + cataract group was $20.05 \pm 9.35$ $\mathrm{mmHg}$ and $19.45 \pm 5.75 \mathrm{mmHg}$, respectively $(p=0.74)$. Baseline characteristics of the patients have been listed in Table 1. The two groups were comparable in terms of average age, male: female ratio, type of glaucoma, and baseline mean deviation. On average, the first needling intervention was performed at 136 days after surgery. There was no significant difference between the two surgical groups in terms of timing (126 days in XEN + cataract surgery group vs 158 in XEN alone group, $p=0.30$ ).

Average RNFL thickness was significantly more in XEN + cataract group $(p=0.007)$. Mean IOP at day 1 was significantly lower in XEN alone group $(p=0.001)$ but no such difference was noted between the two groups at week $1(p=0.18)$. As previously reported, needling was performed in 55 eyes (37\%): 36 eyes (24.2\%) required one, 14 eyes $(9.4 \%)$ two, and 5 eyes $(3.4 \%)$ three needling interventions [5]. In all, 18 eyes (45\%) of XEN alone group vs. $37(34 \%)$ of XEN + cataract eyes required needling. There was no statistically significant association between the type of surgery and rates of needling $(p=0.22)$.

\section{XEN alone group}

The mean IOP at day 1 and week 1 was $9.70 \pm 5.43$ and $10.33 \pm 4.41 \mathrm{mmHg}$, respectively. A statistically significant association was noted between day 1 IOP and probability of needling intervention (OR, 1.14; 95\% CI, 1.02-1.28; $p=$ 0.02 ) ; eyes with lower IOP on day 1 were less likely to require needling during their follow-up. A similar association (OR, 1.15; 95\% CI, 1.06-1.26; $p=0.001$ ) was also observed between day 1 IOP in and number of needling interventions. Patients with lower IOP on day 1 were likely to require fewer needling interventions (Fig. 1a). No significant association was noted between probability or number of needling and IOP at week 1 ( $p=0.163,0.184$, respectively). Probability and number of needling were also not affected by age, the type of glaucoma (POAG or PEXG), glaucoma severity (MD and average RNFL thickness), and number of preoperative anti-glaucoma medications (Table 2).

\section{XEN + cataract group}

The mean IOPs at day 1 and week 1 were $13.75 \pm 7.52$ and $11.81 \pm 5.36 \mathrm{mmHg}$, respectively. Contrary to XEN alone group, no significant association was noted between early postoperative IOP and probability or number of needling (day 1: OR, 1.02; 95\% CI, 0.97-1.08, $p=0.376$, Fig. 1b; week 1: OR, $1.05 ; 95 \%$ CI, $0.95-1.15, p=0.328)$. Age, type of glaucoma, glaucoma severity, and number of preoperative anti-glaucoma medications had a statistically insignificant association with probability and number of needling (Table 2).

The analysis was repeated considering one random eye per subject (29 eyes of 29 patients in XEN alone group and 84 eyes of 84 patients in XEN + cataract group). The results were similar with day 1 IOP being the only factor significantly associated with both the probability of needling (OR: $1.20,95 \%$ CI: $1.01-1.42, p=0.04$ ) and the number of needlings (OR: $1.23,95 \% \mathrm{CI}: 1.05-1.55, p=0.01$ ) in the XEN alone group.

\section{Discussion}

Meticulous postoperative care is essential for long-term success of glaucoma surgery. Early identification of risk factors for failure can enhance outcomes. The predictive value of early postoperative IOP has been proven by various studies on eyes after trabeculectomy, deep 
Table 2 Factors associated with needling

\begin{tabular}{|c|c|c|c|}
\hline Association between & $\begin{array}{l}\text { XEN alone Odds ratio, } \\
95 \% \text { CI ( } \mathrm{p} \text { value) }\end{array}$ & $\begin{array}{l}\mathrm{XEN}+\text { cataract Odds } \\
\text { ratio, 95\% CI ( } \mathrm{p} \text { value) }\end{array}$ & $\begin{array}{l}\text { Overall Odds ratio, } 95 \% \\
\text { CI ( } p \text { value) }\end{array}$ \\
\hline \multicolumn{4}{|c|}{ Requirement of needling and- } \\
\hline Day 1 IOP & $1.14,1.02-1.28(0.020)$ & $1.02,0.97-1.08(0.376)$ & $1.03,0.98-1.08(0.215)$ \\
\hline 1 week IOP & $1.16,0.93-1.45(0.184)$ & $1.05,0.95-1.15(0.328)$ & $1.06,0.97-1.17$ (0.179) \\
\hline Age & $1.00,0.93-1.07(0.910)$ & $0.99,0.95-1.04(0.983)$ & $1.00,0.96-1.03(0.986)$ \\
\hline $\begin{array}{l}\text { Type of glaucoma } \\
\text { (POAG) }\end{array}$ & $0.60,0.14-2.42(0.473)$ & $1.20,0.49-2.96(0.683)$ & $0.99,0.46-2.11(0.990)$ \\
\hline $\begin{array}{l}\text { Type of glaucoma } \\
\text { (PEXG) }\end{array}$ & $1.69,0.40-7.14(0.471)$ & $0.90,0.36-2.24(0.826)$ & $1.05,0.49-2.27(0.889)$ \\
\hline $\begin{array}{l}\text { Number of preoperative } \\
\text { medications }\end{array}$ & $1.18,0.84-1.64(0.326)$ & $0.98,0.81-1.19(0.906)$ & $1.17,0.85-1.61(0.307)$ \\
\hline Baseline mean deviation & $1.02,0.87-1.20(0.749)$ & $0.99,0.91-1.09(0.994)$ & $1.00,0.93-1.08(0.881)$ \\
\hline $\begin{array}{l}\text { Average RNFL } \\
\text { thickness }\end{array}$ & $1.00,0.95-1.04(0.991)$ & $0.97,0.94-1.00(0.173)$ & $0.98,0.95-1.00(0.144)$ \\
\hline \multicolumn{4}{|l|}{ Number of needlings and- } \\
\hline Day 1 IOP & $1.15,1.06-1.26(0.001)$ & $1.01,0.97-1.06(0.435)$ & $\begin{array}{l}0.02,-0.01-0.06 \\
(0.167)\end{array}$ \\
\hline 1 week IOP & $1.16,0.93-1.44(0.163)$ & $1.03,0.94-1.12(0.440)$ & $1.05,0.97-1.14(0.209)$ \\
\hline Age & $0.98,0.94-1.04(0.632)$ & $1.00,0.96-1.05(0.976)$ & $1.00,0.96-1.04(0.787)$ \\
\hline $\begin{array}{l}\text { Type of glaucoma } \\
\text { (POAG) }\end{array}$ & $0.79,0.22-2.81(0.726)$ & $1.16,0.52-2.58(0.706)$ & $1.04,0.53-2.03(0.905)$ \\
\hline $\begin{array}{l}\text { Type of glaucoma } \\
\text { (PEXG) }\end{array}$ & $1.11,0.32-3.85(0.862)$ & $0.81,0.36-1.83(0.622)$ & $0.89,0.45-1.74(0.738)$ \\
\hline $\begin{array}{l}\text { Number of preoperative } \\
\text { medications }\end{array}$ & $1.01,0.67-1.52(0.963)$ & $1.00,0.71-1.39(0.984)$ & $1.04,0.81-1.33(0.760)$ \\
\hline Baseline mean deviation & $1.01,0.86-1.18(0.868)$ & $1.01,0.93-1.11(0.698)$ & $1.06,0.94-1.09(0.684)$ \\
\hline $\begin{array}{l}\text { Average RNFL } \\
\text { thickness }\end{array}$ & $0.99,0.96-1.03(0.982)$ & $0.97,0.94-1.00(0.168)$ & $0.98,0.96-1.00(0.158)$ \\
\hline
\end{tabular}

$C I$ confidence interval, IOP intraocular pressure, $P O A G$ primary open-angle glaucoma, $P E X G$ pseudoexfoliative glaucoma; $R N F L$ retinal nerve fiber layer

Bold letters denote statistically significant values sclerectomy, and selective laser trabeculoplasty [6-8]. In the current study, we extended this hypothesis to the XEN implant and analyzed if early postoperative IOP provided meaningful information about the future course of events. The rate of needling was used as a surrogate measure of determining the effect of early postoperative IOP on success rates of XEN implant.

This study demonstrated that in patients undergoing standalone XEN implantation who had a day 1 IOP of $>20$ $\mathrm{mmHg}$ the probability of needling was as high as $80 \%$. This number decreased to $\sim 35 \%$ if day 1 IOP was $<10 \mathrm{mmHg}$ and to $20 \%$ if $<5 \mathrm{mmHg}$. Higher post operative day $1 \mathrm{IOP}$ not only predisposed to a higher probability of needling but also to a higher number of needling interventions. These results have important implications for the postoperative management of XEN, suggesting that surgeons should not wait too long to intervene via needling if IOP in the early postoperative period is in the high teens or above. However, as no intervention is risk-free, the benefit of needling should always be weighed against possible complications, such as damage to the implant, leak through the needle entry site, infection, hypotonic maculopathy, and choroidal detachment [5, 9-11].

No significant association was observed between day 1 IOP and needling interventions in XEN + cataract group, however we found that mean IOP on day 1 in XEN alone group was significantly lower compared to XEN + cataract group $(p=0.0012)$. This lower day 1 IOP translated into significantly better outcomes at 1 year in the XEN alone group with $81 \%$ eyes achieving complete success versus $56.1 \%$ in $\mathrm{XEN}+$ cataract group $(p=0.04)$ [5]. This observation reinforces the importance of achieving lower IOPs in the early postoperative period.

Increased IOP in the early postoperative period stretches the bleb, which incites fibroblast proliferation and fibrosis. This, in turn, increases the IOP further. This vicious cycle should be interrupted at the earliest to prevent failure of the surgery [12].

In eyes undergoing trabeculectomy the subconjunctival and episcleral tissues have been dissected intraoperatively, thus offering less resistance to aqueous outflow in the 
immediate postoperative period. On the other hand, in eyes with XEN implantation there is minimal manipulation of subconjunctival and episcleral tissues and therefore may require needling in the immediate postoperative period. The cause of high IOP on day 1 after surgery has been postulated to be due to blockage of the XEN implant by Tenon, blood, or exudates but has not been studied in detail $[2,13]$.

Based on the results of this study, we have modified our surgical technique. First, at the end of the surgery, the XEN implant is carefully manipulated through the conjunctiva with a blunt instrument (vitreous spatula, cotton tip, etc.) to ensure that implant is freely mobile with no Tenon blocking the lumen of the tube. Second, balanced salt solution is injected into the anterior chamber to verify the patency of the implant via formation of a diffuse bleb. In our experience, these two minor modifications have resulted in more patients presenting with a single digit IOP on first postoperative day.

The type or severity of glaucoma did not have any influence on the probability or number of needling interventions required further emphasizing the importance of wound modulation process over other factors in predicting postoperative outcomes. At last, identifying these predictors helps the patient as well as the surgeon to be better informed and prepared for future interventions.

A limitation of our study is the lack of morphological assessment of bleb. Bleb appearance has been shown to be a predictor of bleb function and necessity of needling [14]. A second limitation is the fact that surgeries were performed by two different surgeons and the decision for needling was at surgeon's discretion with no standardized protocol.

In summary, we have demonstrated that postoperative day 1 IOP is a predictor for needling in patients undergoing XEN implantation alone. The probability of needling doubles if $\mathrm{IOP}>10 \mathrm{mmHg}$ is recorded on postoperative day 1 and quadruples if IOP recorded is $>20 \mathrm{mmHg}$. Type of glaucoma and glaucoma severity did not influence the probability of needling or number of needling interventions required.

\section{Summary}

\section{What was known before}

- XEN is the only FDA-approved MIGS device, which drains into subconjunctival space and results in bleb formation.

- Needling is the most frequently reported adverse event after XEN implantation.

\section{What this study adds}

- IOP on the postoperative day 1 can provide meaningful insight into surgical outcomes.
- $\mathrm{IOP}>20 \mathrm{~mm} \mathrm{Hg}$ on day 1 after surgery increases the probability of needling to $80 \%$.

- This relationship between day 1 IOP and needling rates were observed only in XEN alone group. No similar relationship was observed in XEN + cataract group.

Funding Midha N: none; Rao HL: Pfizer (C), Santen (C), Cipla (C); Mermoud A: Santen (C), Sensimed (C), Topcon (S), Alcon (S), Allergan (S); Mansouri K: Santen (C), Sensimed (C), Topcon (S), Alcon (S), Allergan (S), Optovue (S), ImplanData (C). Supported in part by the Swiss Glaucoma Research Foundation, Lausanne, Switzerland.

\section{Compliance with ethical standards}

Conflict of interest The authors declare that they have no conflict of interest.

\section{References}

1. Vinod K, Gedde SJ. Clinical investigation of new glaucoma procedures. Curr Opin Ophthalmol. 2017;28:187-93.

2. Chaudhary A, Salinas L, Guidotti J, et al. XEN gel implant: a new surgical approach in glaucoma. Expert Rev Med Devices. 2018;15:47-59.

3. Filippopoulos T, Hanna E, Chen TC, Grosskreutz CL, Jakobiec FA, Pasquale LR. Correlation of filtration bleb morphology with histology. Int Ophthalmol Clin. 2009;49:71-82.

4. Yook E, Vinod K, Panarelli JF. Complications of micro-invasive glaucoma surgery. Curr Opin Opthalmol. 2018;29:147-54.

5. Mansouri K, Guidotti J, Rao HL et al. Prospective evaluation of standalone XEN gel implant and combined phacoemulsificationXEN gel implant surgery: 1-Year results. J Glaucoma. 2018;27:140-47.

6. Rong SS, Meng HL, Fan SJ, et al. Can intraoperative intraocular pressure during primary trabeculectomy predict early postoperative pressure? J Glaucoma. 2014;23:653-7.

7. Shaarawy T, Flammer J, Smits G, et al. Low first postoperative day intraocular pressure as a positive prognostic indicator in deep sclerectomy. Br J Ophthalmol. 2004;88:658-61.

8. Johnson PB, Katz LJ, Rhee DJ. Selective laser trabeculoplasty: predictive value of early intraocular pressure measurements for success at 3 months. Br J Ophthalmol. 2006;90:741-3.

9. Rotchford AP, King AJ. Needling revision of trabeculectomies bleb morphology and long-term survival. Ophthalmology. 2008; 115:1148-.e4.

10. Alwitry A, King AJ, Vernon SA. Successful combined cataract surgery and drainage of needling-induced chronic ciliochoroidal detachment. Eye. 2005;19:478-80.

11. Broadway DC, Bloom PA, Bunce C, et al. Needle revision of failing and failed trabeculectomy blebs with adjunctive 5-fluorouracil: survival analysis. Opthalmology. 2004;111:665-73.

12. Wang JH, Thampatty BP, Lin JS, et al. Mechanoregulation of gene expression in broblasts. Gene. 2007;391:1-15.

13. Pinto Ferreira N, Abegão Pinto L, Marques-Neves C. XEN gel stent internal ostium occlusion: ab-interno revision. J Glaucoma. 2017;26:e150-e152.

14. Lee YS, Wu SC, Tseng HJ, et al. The relationship of bleb morphology and the outcome of needle revision with 5fluorouracil in failing filtering bleb. Medicine (Baltimore). 2016;95:e4546. 